\title{
Linking star formation and environment in supercluster galaxies
}

\author{
M.E. Gray ${ }^{1}$, C. Wolf ${ }^{2}$, K. Meisenheimer ${ }^{3}$, A. Taylor ${ }^{4}$, S. Dye ${ }^{5}$, \\ A. Borch ${ }^{3}$ and M. Kleinheinrich ${ }^{3}$ \\ ${ }^{1}$ School of Physics and Astronomy, University of Nottingham, Nottingham NG7 1GW, UK \\ email: Meghan.Gray@nottingham.ac.uk \\ ${ }^{2}$ Department of Physics, University of Oxford, Keble Road, Oxford OX1 3RH, UK \\ ${ }^{3}$ Max-Planck-Institut für Astronomie, Königstuhl 17, D-69117, Heidelberg, Germany \\ ${ }^{4}$ Institute for Astronomy, University of Edinburgh, Blackford Hill, Edinburgh EH9 3HJ \\ ${ }^{5}$ Astrophysics Group, Blackett Lab, Imperial College, Prince Consort Road, London SW7 2BW
}

\begin{abstract}
We present a unique multiwavelength dataset of the Abell 901/902 supercluster at $z=0.16$. The environment of the structure is well characterized by weak lensing analysis, galaxy dynamics, and X-ray imaging, and we examine how galaxy properties correlate with these measures of environment. We examine the star-formation-density relation and find a surface mass density threshold above which star-formation in supercluster galaxies is truncated, and that this transformation affects primarily the faint end of the star-forming galaxy population (down to $M^{*}+6$ ). In addition, we present a preliminary dynamical analysis of the spectroscopic sample ( $n \sim 300$ galaxies), revealing a bimodal distribution of galaxy velocities and significant substructure.
\end{abstract}

\section{The dark matter environment}

The influence of environment on the structure and star-formation properties of galaxies is of key importance in the quest to understand the physical process responsible for galaxy evolution. Star-formation-density relations have been observed in the SDSS and 2dF surveys (Lewis et al. 2002, Gómez et al. 2004, Balogh et al. 2004) and in the infall region around a $z=0.4$ cluster (Kodama et al. 2001). In each of these studies, 'environment' has been characterized by local galaxy density. By contrast, here we use gravitational lensing to examine the variation of star-formation activity directly with the dark matter environment in a low-redshift supercluster system.

The A901/902 supercluster was one of 4 fields targeted by the COMBO-17 band survey (Wolf et al. 2003, 2004). The structure, comprised of 3 clusters and associated filametary structure all at $z=0.16$ is fully contained in the $0.5^{\circ} \times 0.5^{\circ}$ field-of-view of the ESO Wide-Field Imager (WFI). The 5 broad bands and 12 medium bands ensure accurate photometric redshifts and spectral classifications in a complete sample down to $R=24$. Furthermore, high-quality $R$-band imaging with sub-arcsecond seeing provides the basis for a $2 \mathrm{D}$ weak lensing analysis of the region (Gray et al. 2002) as well as the first attempt at a 3D lensing inversion (Taylor et al. 2004).

Having characterized the distribution of dark matter in the field with weak lensing, we investigate correlations between the location of galaxies in dense environments and their degree of star-formation activity. Using the photometric redshift catalogue we are able to precisely isolate galaxies from a $4 \times 4 h^{-1} \mathrm{Mpc}$ region around the supercluster in the range $0.15<z<0.18$. We measure rest-frame photometric quantities from PEGASE template fits to the 17-band photometric data, and divide supercluster galaxies into two 

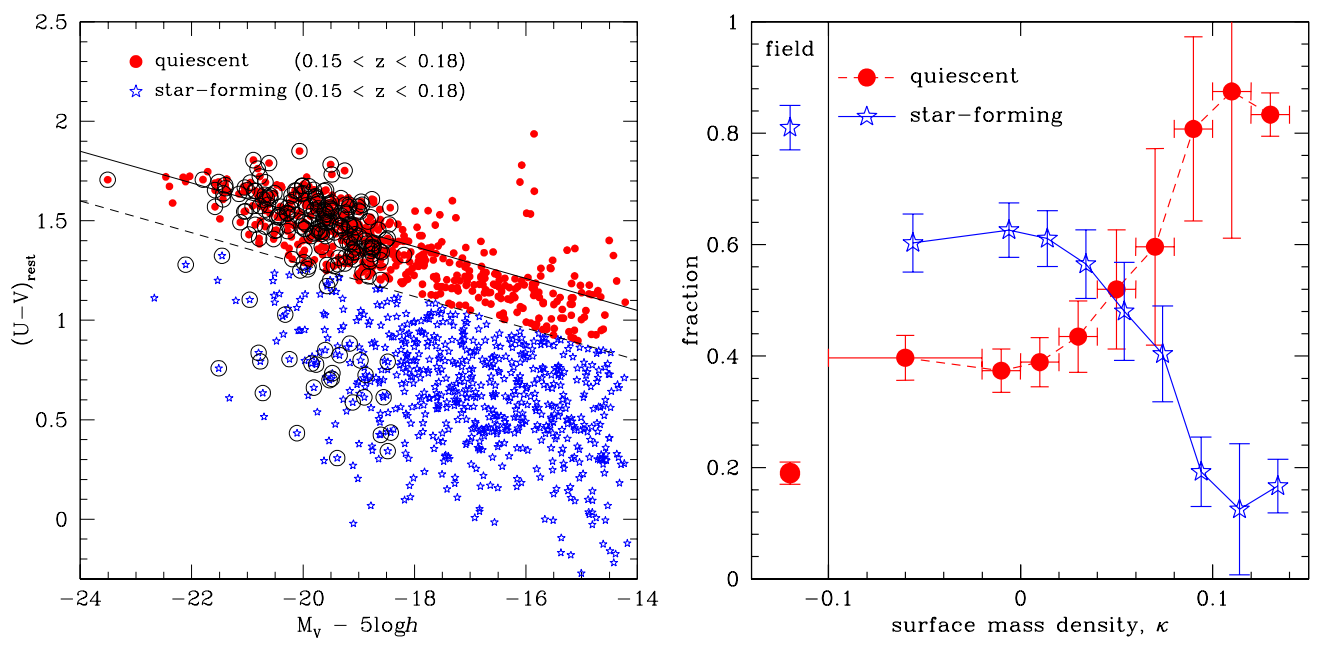

Figure 1. Left: The rest-frame $U-V$ vs $M_{V}$ colour-magnitude diagram for supercluster galaxies with $0.15<z_{\text {phot }}<0.18$. Open circles indicate galaxies for which $2 \mathrm{dF}$ spectra have been obtained. Right: The SF- $\kappa$ relation for the A901 supercluster. A dramatic change in relative abundance of each galaxy type at a given density is seen as the proportion of star-forming galaxies declines and quiescent galaxies become more dominant in dense regions. The symbols at left show the corresponding fractions reached in the field, obtained from an identical redshift slice within two blank COMBO-17 fields (Gray et al. 2004).

classes ('quiescent' and 'star-forming') based on deviation blueward of the $(U-V)_{\text {rest }}$ vs. $M_{V}$ colour-magnitude sequence (Fig. 1a).

We sample the weak gravitational lensing mass-map of Gray et al. (2002) at the location of each galaxy in order to use mass as a direct measurement of environment for the first time. We observe (Gray et al. 2004):

- clear evidence of segregation by type, with the highest density regions populated almost exclusively by galaxies classified according to their rest-frame $U-V$ colours as quiescent;

- a threshold surface mass density from lensing, $\kappa=0.05$, above which star-formation activity is rapidly suppressed (Fig. 1b);

- that this abrupt transformation affects primarily the faint end of the star-forming galaxy population (down to $M^{*}+6$ ) and occurs at a local surface number density that is an order-of-magnitude above the density of the field;

- that when only galaxies brighter than $M_{V}^{*}+1$ are considered the trends with environment remain, but are more gradual and extend beyond $2 h^{-1} \mathrm{Mpc}$ radius.

\section{The spectroscopic sample}

The A901/902 field was observed with the 2dF spectrograph on the AAT over two observing campaigns: one night in March 2002 (using the 1200B grating) and one night in March 2003 (using the 600V grating). Targets having $R<20$ were selected in the first instance by COMBO-17 redshift indicating likely cluster membership. Additional fibres were allocated to other objects in the field where possible. Even with multiple fibre configurations (one per night) the limitations placed on fibre allocation by the crowded nature of the field meant that the sampling was incomplete and that several of the brightest cluster members were missed. 

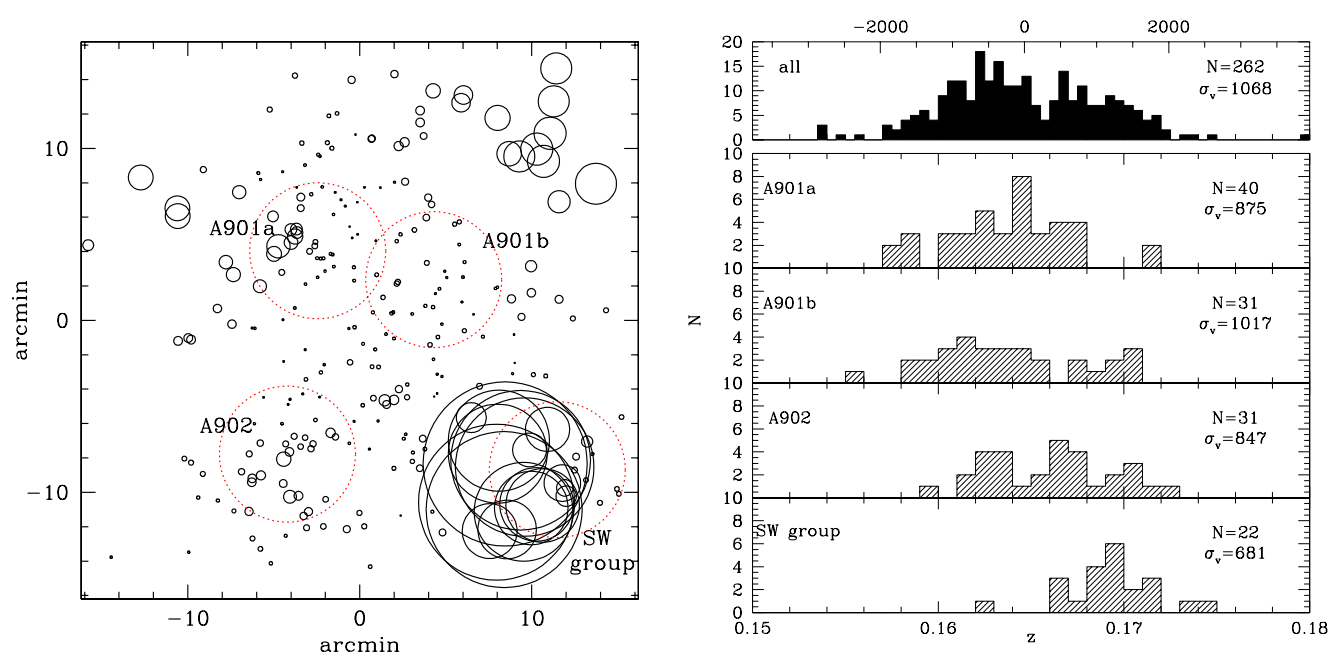

Figure 2. Left: The results of a Dressler-Shectman test on supercluster velocities, revealing significant substructure. The large number of large circles in the SW corner indicate a group that is distinct relative to the three main clusters both spatially and kinematically. Right: Redshift histograms and velocity dispersions for the full sample (top) and the three clusters and one group selected in regions indicated by the large dashed circles in the left-hand image. The full sample hints at some degree of bimodality, but the spatially-selected structures do not segregate in redshift space in a straightforward manner (with the exception of the SW group centred around $z=0.17$ ).

Galaxy redshifts were determined and cross-checked according to two methods: (a) fitting of the prominent $\mathrm{H}$ and $\mathrm{K}$ absorption features by eye, and (b) cross-correlation with known templates. In total 373 galaxy redshifts were obtained, 263 of which were in the range of interest $0.15<z<0.18$. Of that sample, 43 were observed during both campaigns. A Dressler-Shectman (1988) test shows significant substructure in the field, particularly a group to the SW of the main structure (Fig. 2a). Monte Carlo simulations using randomized velocities show that the probability of producing this level of substructure by chance is $P<0.0001$. Redshift histograms for the full field and spatially selected galaxies around the known mass peaks and galaxy overdensities reveal distinct components, particularly a group at $z=0.16$ southwest of the three main clusters.

\section{Further work}

In addition to the aforementioned multicolour and spectroscopic dataset, we are also in the process of analyzing a deep 90ks XMM of the field. A rudimentary first-look analysis shows extensive diffuse emission from all three clusters as well as the SW group, and a large number of point sources which appear to trace the same structure. The relative prominence of the extended emission around each cluster appears contrary to naive predictions from either the weak lensing map or the galaxy distributions. Interestingly, the largest extended peak in the X-ray map corresponds to A901b: in this cluster the galaxy distribution is highly elongated and is turn misaligned with the mass peak. Thus we find that as we dissect the supercluster layer by layer, each measure of the 'environment' yields a different picture of this complex structure.

In summary, our goal is to employ the full dataset to accurately characterize the environmental influences that may be acting on the infalling galaxies, whether by the 
influence of the cluster dark matter, hot X-ray gas, or high galaxy number density. We hope to disentangle which interactions (whether between the galaxy and the cluster gravitational field, the galaxy and the intracluster medium, or galaxy-galaxy interactions) are responsible for the observed transformation of photometric and morphological properties.

\section{References}

M. Balogh et al. 2004 MNRAS 348, 1355

A. Dressler \& S. Shectman 1988 AJ 95, 985

M. Gray, A. Taylor, K. Meisenheimer, S. Dye, C. Wolf, E. Thommes 2002 ApJ 568

M. Gray, C. Wolf, K. Meisenheimer, A. Taylor, S. Dye, A. Borch, M. Kleinheinrich 2004 MNRAS 437, 73L

P. Gómez et al. 2003 ApJ 584, 210

T. Kodama, I. Smail, F. Nakata, S. Okamura, R.G. Bower 2001 ApJ 562, 9

I. Lewis et al. 2002 MNRAS 334, 673

A.N. Taylor, D.J. Bacon, M.E. Gray, C. Wolf, K. Meisenheimer, S. Dye, A. Borch, M. Kleinheinrich, Z. Kovacs, L. Wisotzki 2004, MNRAS, submitted (astro-ph/0402095)

C. Wolf, K. Meisenheimer, H.-W. Rix, A. Borch, S. Dye, M. Kleinheinrich 2003 A\&A 401, 73

C. Wolf, K. Meisenheimer, M. Kleinheinrich, A. Borch, S. Dye, M. Gray, L. Wisotzki, E. F. Bell, H.-W. Rix, A. Cimatti, G. Hasinger, G. Szokoly 2004 A\&A, submitted (astro-ph/0403666) 